\title{
Comparison of the Absorb bioresorbable vascular scaffold to the Xience durable polymer everolimus-eluting metallic stent in routine clinical practice: a propensity score-matched analysis from a multicenter registry
}

\author{
Bartłomiej Orlik ${ }^{1,2}$, Krzysztof Milewski ${ }^{1,2}$, Kamil Derbisz ${ }^{1}$, Michał Jelonek ${ }^{1}$, Patrycja Chrząszcz $^{1}$, Sonia Beil ${ }^{1}$, \\ Adam Młodziankowski³ , Wojciech Picheta ${ }^{4}$, Piotr P. Buszman ${ }^{1}$, Paweł E. Buszman ${ }^{1}$ \\ ${ }^{1}$ Center for Cardiovascular Research and Development, American Heart of Poland, Katowice, Poland \\ ${ }^{2} 10^{\text {th }}$ Department of Invasive Cardiology, Electrophysiology and Electrostimulation, Tychy, Poland \\ $3^{\text {th }}$ Department of Invasive Cardiology and Angiology, Mielec, Poland \\ ${ }^{4}$ Department of Internal Medicine, District Hospital, Myszkow, Poland
}

Adv Interv Cardiol 2018; 14, 2 (52): 149-156

DOI: https://doi.org/10.5114/aic.2018.76406

\begin{abstract}
A bstract
Introduction: Most clinical trials related to bioresorbable vascular scaffold (BVS) technology are limited to a highly selected patient population.

Aim: To evaluate early and long-term clinical outcomes of the Absorb everolimus-eluting BVS compared to the everolimus-eluting metallic XIENCE $V$ stent in routine clinical practice.

Material and methods: This is a multicenter, retrospective propensity score-matched comparative study, comprising 76 patients treated with a bare metal stents (BMS) and 501 with a XIENCE stent. Patients included in the study had stable and unstable angina and both types of myocardial infarction (STEMI and NSTEMI) as an indication for intervention and at least one significant de novo lesion in native coronary arteries. The primary endpoint was major adverse cardiovascular event (MACE), defined as death, myocardial infarction (MI), or target vessel revascularization (TVR).

Results: Median follow-up was 400 days in both groups. After propensity score matching for patient baseline characteristics, only higher rate of predilatation, predominantly treated left anterior descending artery (LAD) and lower number of used stents in the BVS group remained statistically significant. After adjustment there was no difference in type of treated lesions. The MACE rate did not differ between BVS and drug-eluting stents (DES) groups (7.2\% vs. $11.15 \%$, respectively; $p=0.17$ ). The TVR was $2.9 \%$ in both groups. Except in the periprocedural period, there were no deaths or MI in the BVS group. There was no stent thrombosis in either studied group.

Conclusions: In routine clinical practice throughout long-term follow-up, clinical outcomes of patients who successfully received the Absorb BVS did not differ from those of patients who received the Xience stent. Longer follow-up data are required to determine whether these findings will persist beyond one year.
\end{abstract}

Key words: acute coronary syndrome, drug-eluting stent, stable angina, bioresorbable vascular scaffold, coronary percutaneous intervention.

\section{Introduction}

The introduction of drug-eluting stents (DES) to the treatment of atherosclerotic lesions in the coronary territory significantly improved the clinical outcomes when compared to bare metal stents (BMS). Drug-eluting stents allow controlled release of antiproliferative drugs that effectively reduce neointimal hyperplasia. However, due to their permanent nature they do not eliminate the risk of late clinical events including very late restenosis, neoatherosclerosis, late stent thrombosis and stent fractures $[1,2]$. Theoretically, a promising alternative is a bioresorbable vascular scaffold (BVS), which elutes an antiproliferative compound in a similar way to metallic DES and then resorbs naturally, leaving no permanent scaffold, allowing the vessel to restore its physiological functions and theoretically eliminate the risk of late clinical events [3]. 
The Absorb BVS (Abbott Vascular, Santa Clara, USA) elutes everolimus, similarly to the XIENCE $V$, a second generation DES, but the platform consists of a fully biodegradable polymer instead of a cobalt-chromium platform.

Although initial clinical trials with BVS showed promising results, the long-term observations revealed several limitations, including high risk of target vessel related myocardial infarction and device thrombosis [4, 5]. As these outcomes are mostly related to a highly selected patient population, it is important to test the effects of BVS implantation in routine clinical practice including patients with stable angina and those presenting with acute coronary syndromes.

\section{Aim}

The aim of this study was to evaluate early and longterm clinical outcomes of the Absorb everolimus-eluting BVS (Abbott Vascular, Santa Clara, USA) compared to the everolimus-eluting metallic XIENCE V DES (Abbott Vascular) in routine clinical practice.

\section{Material and methods}

\section{Study design and population}

This is a multicenter, retrospective propensity scorematched comparison of patients with coronary artery disease who underwent $\mathrm{PCl}$ with BVS or Xience $\mathrm{V}$ stent implantation between July 2013 and June 2014 in eight Departments of American Heart of Poland (AHP). Seventy-six patients who received an Absorb BVS (BVS group) and 501 consecutive subjects with implanted Xience stent (DES group) and met described below inclusion criteria were selected for analysis.

The main inclusion criteria were adult patients with stable angina (SA), unstable angina (UA), non-ST segment elevated myocardial infarction (NSTEMI) or ST segment elevated myocardial infarction (STEMI), with at least one significant lesion in native coronary arteries.

Patients in whom stents other than those being tested were implanted were excluded from the study. Concurrent use of the same type of stent (BVS or Xience V) was permitted. Subjects with contradictions for prolonged dual antiplatelet therapy (DAPT), in cardiogenic shock, with left ventricle ejection fraction (LVEF) $<35 \%$, chronic kidney disease with eGFR $<30 \mathrm{ml} / \mathrm{min} / \mathrm{m}^{2}$ or life expectancy $<12$ months were excluded from the registry.

\section{Procedural characteristics}

Loading doses of clopidogrel or ticagrelor with aspirin (as recommended by ESC guidelines) were given to all patients before or immediately after percutaneous procedures. During the procedure patients received $100 \mathrm{IU} /$ kg i.v. of unfractionated heparin, which was titrated to maintain an ACT > $300 \mathrm{~s}$. Use of GP IIb/IIla receptor inhibitors was at the operator's discretion. All patients were advised to continue taking aspirin indefinitely and clopidogrel or ticagrelor for at least 12 months.

Percutaneous coronary intervention was performed using standard techniques. The decision to use a particular stent type or procedural techniques was made independently by the operator.

\section{Study endpoints and definitions}

Due to the observatory nature of this study no preliminary hypotheses were generated. The primary endpoint of the study was major adverse cardiovascular event (MACE), defined as death of any cause, myocardial infarction (MI) [6], including periprocedural MI and target vessel revascularization (TVR). Target vessel revascularization was defined as any symptomatic repeated revascularization (percutaneous or surgical) of a previously stented coronary artery. Device thrombosis (stent/scaffold) was defined with reference to the Academic Research Consortium Definitions of Stent Thrombosis [7].

\section{Study device}

The Absorb BVS (Abbott Vascular, Santa Clara, USA) is a fully bioresorbable vascular scaffold which consists of a fully biodegradable poly-L-lactide (PLLA) platform covered by an amorphous matrix of poly-D,L-lactide (PDLLA) and everolimus. It is gradually resorbed in the process of hydrolysis to non-inflammatory products, such as $\mathrm{CO}_{2}$ or $\mathrm{H}_{2} \mathrm{O}$ [3]. The average strut thickness is $157 \mu \mathrm{m}$. Eighty percent of drug release occurs within 30 days.

The Xience V (Abbott Vascular, CA, USA) is a cobaltchromium stent coated with a non-erodible fluoropolymer loaded with $100 \mu \mathrm{g} / \mathrm{cm}^{2}$ of everolimus. The thickness of the metallic struts and coating combined is approximately $90 \mu \mathrm{m}(81 \mu \mathrm{m}$ for the stent and $7.8 \mu \mathrm{m}$ for the polymer $\approx 90$ ) [8]. The antiproliferative drug concentration and release profile are similar in both stents.

\section{Data collection and follow-up}

Clinical and procedural data were collected based on an anamnesis with patients and hospital electronic system containing all discharge information. The sites possess quality assurance ISO 9001 certificates and are frequently audited by the National Health Fund. The data on the incidence of other adverse events were collected by telephone interview with the patient or with her/his family and all outcomes of interest were confirmed with hospital discharge files.

\section{Statistical analysis}

Normally distributed parametric data were expressed as the mean and standard deviation. In cases of skewed distribution, data were expressed as median and interquartile range. Nonparametric data were presented as an absolute number and percentage. 95\% confidence 
intervals (Cls) and relative risk (RR) for follow-up results were calculated. The parametric variables between the groups were compared using Student's $t$-test, while a $\chi^{2}$ test was used for comparison of nonparametric data. If any analyzed group was smaller than 5 observations, the Fisher two-tailed exact test was used to evaluate statistical significance. A $p$-value of $<0.05$ was considered to indicate statistical significance. Because of the nonrandomized nature of the study, propensity score analysis was used to adjust for differences in patients' baseline characteristics. Additionally, the Kaplan-Meier method was performed to estimate the survival curve in both groups. All statistical analyses were performed using Statistica, version 12.0.

\section{Results}

\section{Study population}

Baseline clinical and demographic characteristics of both groups are presented in Table I. In both groups the patients were predominantly men (78\% and 68\% in BVS and DES group, respectively, $p=0.07$ ). The prevalence of cardiovascular risk factors, such as hypertension, diabetes and smoking, was similar in both groups.

Patients in the DES group were slightly older than the BVS group (60 vs. 65 years; $p<0.001$, respectively). The main indication for $\mathrm{PCl}$ in both study groups was $U A$, but stable angina was significantly more prevalent in the BVS group ( $34 \%$ vs. $23 \% ; p=0.04)$. There was a higher incidence of myocardial infarction (NSTEMI and STEMI) in the DES group.

The most prominent difference between the groups was presence of multivessel disease (defined as the pres- ence of greater than or equal to $70 \%$ luminal diameter stenosis in two or more major epicardial arteries), which was present in $38 \%$ of patients in the BVS group and $60 \%$ of those with DES $(p<0.001)$.

Propensity score matching of baseline clinical and demographic data resulted in bias reduction in most of the parameters (data presented in Table II). Only previous $\mathrm{PCl}$ procedure remained significantly different between studied groups.

\section{Procedural data and short-term results}

Unadjusted procedural data are presented in Table III. The mean stent diameter and length were comparable between BVS and DES groups (3.0 vs. $2.9 \mathrm{~mm} ; p=0.26$ and 22.1 vs. $21.5 \mathrm{~mm} p=0.54$, respectively). In the DES group, a significantly higher number of stents was needed during the procedure of interest (1.0 vs. 1.26, $p=0.001)$. The predilatation rate was significantly higher in the BVS group ( $76 \%$ vs. $38 \%, p<0.001)$, while postdilatation was performed at a similar rate (36\% vs. $40 \%$, $p=0.54)$. Stents were more frequently implanted in the left anterior descending artery (LAD) in the BVS group than in the DES group (59\% vs. $46 \% ; p=0.03$ ). In the BVS group, the type of treated lesion was mostly A or B1 (76\%), whereas in the DES group there was an almost equal proportion of lesions of type A/B1 and B2/C (54\% vs. $46 \%$, respectively). There were no differences with regard to incidence of ostial and bifurcated lesions. In both groups, there were no cases of intravascular ultrasound imaging or optical coherence tomography. There were no differences with regard to DAPT regimen.

After propensity score matching, only higher rate of predilatation, predominantly treated LAD and lower num-

Table I. Unadjusted baseline clinical and demographic data

\begin{tabular}{|c|c|c|c|}
\hline Parameter & BVS $(n=76)$ & DES $(n=501)$ & $P$-value \\
\hline Age [years] & $60 \pm 11.2$ & $65 \pm 9.3$ & $<0.001$ \\
\hline Male & $78 \%$ & $68 \%$ & 0.07 \\
\hline Current smoker & $20 \%$ & $18 \%$ & 0.64 \\
\hline Hypertension & $82 \%$ & $77 \%$ & 0.38 \\
\hline Hyperlipidemia & $54 \%$ & $37 \%$ & 0.006 \\
\hline Diabetes & $30 \%$ & $31 \%$ & 0.96 \\
\hline Previous MI & $28 \%$ & $38 \%$ & 0.07 \\
\hline Previous $\mathrm{PCl}$ & $41 \%$ & $50 \%$ & 0.14 \\
\hline Previous CABG & $1.3 \%$ & $6.6 \%$ & 0.12 \\
\hline Previous stroke & $1.3 \%$ & $4.4 \%$ & 0.33 \\
\hline Peripheral artery disease & $6.6 \%$ & $8.4 \%$ & 0.58 \\
\hline Multivessel disease & $38 \%$ & $60 \%$ & $<0.001$ \\
\hline Left ventricle ejection fraction (\%) & $55 \pm 9.0$ & $52 \pm 8.9$ & 0.005 \\
\hline
\end{tabular}


Table II. Adjusted (after propensity score matching) baseline clinical and demographic data

\begin{tabular}{lccc} 
Parameter & BVS $(n=39)$ & DES $(n=69)$ & $P$-value \\
\hline Age [years] & $60 \pm 11.0$ & $60 \pm 9.0$ & 0.93 \\
\hline Male & $78 \%$ & $67 \%$ & 0.12 \\
\hline Current smoker & $20 \%$ & $29 \%$ & 0.23 \\
\hline Hypertension & $80 \%$ & $83 \%$ & 0.66 \\
\hline Hyperlipidemia & $56 \%$ & $43 \%$ & 0.12 \\
\hline Diabetes & $30 \%$ & $36 \%$ & 0.47 \\
\hline Previous MI & $26 \%$ & $32 \%$ & 0.45 \\
\hline Previous PCl & $38 \%$ & $61 \%$ & 0.006 \\
\hline Previous CABG & $1.5 \%$ & $1.5 \%$ & 1 \\
\hline Previous stroke & $1.5 \%$ & $6 \%$ & 0.36 \\
\hline Peripheral artery disease & $7 \%$ & $4.3 \%$ & 0.71 \\
\hline Multivessel disease & $41 \%$ & $54 \%$ & 0.12 \\
\hline Left ventricle ejection fraction (\%) & $55 \pm 9.2$ & $55 \pm 6.9$ & 0.99
\end{tabular}

Table III. Unadjusted procedural data

\begin{tabular}{|c|c|c|c|}
\hline Parameter & BVS $(n=76)$ & DES $(n=501)$ & $P$-value \\
\hline \multicolumn{4}{|l|}{ Indication for PCl: } \\
\hline SA & $34 \%$ & $23 \%$ & 0.04 \\
\hline UA & $57 \%$ & $52.5 \%$ & 0.5 \\
\hline NSTEMI & $7 \%$ & $15 \%$ & 0.02 \\
\hline STEMI & $3 \%$ & $9 \%$ & 0.09 \\
\hline \multicolumn{4}{|l|}{ Target lesion location: } \\
\hline LAD & $59 \%$ & $46 \%$ & 0.03 \\
\hline$L C x$ & $11 \%$ & $16 \%$ & 0.19 \\
\hline RCA & $21 \%$ & $23 \%$ & 0.68 \\
\hline Other & $9 \%$ & $15 \%$ & 0.17 \\
\hline Left main & $0 \%$ & $7 \%$ & 0.006 \\
\hline \multicolumn{4}{|l|}{ Procedural data: } \\
\hline Predilatation & $76 \%$ & $38 \%$ & $<0.001$ \\
\hline Number of stents & $1-100 \%$ & $\begin{array}{c}1-79 \% \\
2-17 \% \\
3-3 \% \\
4-0.6 \%\end{array}$ & $<0.001$ \\
\hline Stent/scaffold diameter [mm] & $3 \pm 0.4$ & $2.9 \pm 0.5$ & 0.26 \\
\hline Stent/scaffold length [mm] & $22.1 \pm 5.2$ & $21.5 \pm 8.1$ & 0.54 \\
\hline Postdilatation & $36 \%$ & $40 \%$ & 0.54 \\
\hline Type of lesion: & $n=76$ & $n=105$ & \\
\hline $\mathrm{A} / \mathrm{B} 1$ & $76 \%$ & $54 \%$ & 0.009 \\
\hline $\mathrm{B} 2 / \mathrm{C}$ & $24 \%$ & $46 \%$ & 0.01 \\
\hline Ostial & $7 \%$ & $7.6 \%$ & 0.85 \\
\hline Bifurcated & $26 \%$ & $28 \%$ & 0.84 \\
\hline Calcified & $5 \%$ & $6 \%$ & 0.87 \\
\hline
\end{tabular}

values are mean $\pm S D$ or $n(\%)$. 
ber of used stents in the BVS group remained statistically significant. It is important to stress that after adjustment there was no difference in type of treated lesions (Table IV).

In short-term observation, the MACE rate between BVS and DES groups was comparable (1.3\% vs. $1.4 \%$; $p=\mathrm{NS}$, respectively). In the BVS group one cardiac death occurred in the periprocedural period (rupture of the vessel during predilatation), while in the DES group five myocardial infarctions and five deaths were noted within 30 days of follow-up. The differences remained insignificant after adjustment.

\section{Long-term results}

Median follow-up was 400 days in both groups. Before adjustment for patient baseline characteristics, the occurrence of MACE was significantly higher in the DES than the BVS group (24\% vs. $6.6 \%, p=0.011$ ) (Figure 1 ). The difference was predominantly due to higher incidence (though not significant) of repeated revasculariza- tion in the DES group (5.3\% vs. $18 \%, p=0.088$ ). Detailed analysis demonstrated a numerically lower rate of TVR (2.6\% vs. $7 \%, p=0.44)$ and target lesion revascularization (TLR) $(1.3 \%$ vs. $6 \%, p=0.37)$ in the BVS group than in the DES group, although the difference was minor and statistically insignificant. Similarly, survival curves favored the BVS, although the difference was not statistically significant.

After adjustment, the MACE rate between studied groups did not differ significantly (BVS $=7.2 \%$ vs. DES $=$ $11.15 \% ; p=0.17$ ). Repeated revascularization remained a major cause of adverse events, with a TVR rate of $2.9 \%$ in both groups (Figure 2). It is worth emphasizing that, except in the periprocedural period, there were no deaths or myocardial infarctions in the BVS group.

\section{Discussion}

Despite constant development of interventional devices, new DES technologies still have a number of lim-

Table IV. Adjusted (after propensity score matching) procedural data

\begin{tabular}{|c|c|c|c|}
\hline Parameter & BVS $(n=69)$ & $\operatorname{DES}(n=69)$ & $P$-value \\
\hline \multicolumn{4}{|l|}{ Indication for $\mathrm{PCl}$ : } \\
\hline SA & $29 \%$ & $38 \%$ & 0.27 \\
\hline UA & $61 \%$ & $49 \%$ & 0.17 \\
\hline NSTEMI & $7 \%$ & $7 \%$ & 1 \\
\hline STEMI & $3 \%$ & $6 \%$ & 0.68 \\
\hline \multicolumn{4}{|l|}{ Target lesion location: } \\
\hline $\mathrm{LAD}$ & $59 \%$ & $42 \%$ & 0.04 \\
\hline$L C x$ & $12 \%$ & $9 \%$ & 0.57 \\
\hline RCA & $19 \%$ & $19 \%$ & 1 \\
\hline Other & $10 \%$ & $9 \%$ & 0.77 \\
\hline Left main & $0 \%$ & $1.5 \%$ & NS \\
\hline \multicolumn{4}{|l|}{ Procedural data: } \\
\hline Predilatation & $76 \%$ & $39 \%$ & $<0.001$ \\
\hline Number of stents & $1-100 \%$ & $\begin{array}{c}1-81 \% \\
2-13 \% \\
3-4 \% \\
4-1.5 \%\end{array}$ & 0.0002 \\
\hline Stent/scaffold diameter [mm] & $3 \pm 0.4$ & $2.9 \pm 0.5$ & 0.19 \\
\hline Stent/scaffold length [mm] & $22.3 \pm 5.1$ & $22.1 \pm 7.5$ & 0.83 \\
\hline Postdilatation & $36 \%$ & $38 \%$ & 0.74 \\
\hline \multicolumn{4}{|l|}{ Type of lesion: } \\
\hline $\mathrm{A} / \mathrm{B} 1$ & $75 \%$ & $72.5 \%$ & 0.78 \\
\hline $\mathrm{B} 2 / \mathrm{C}$ & $25 \%$ & $27.5 \%$ & 0.54 \\
\hline Ostial & $7 \%$ & $7 \%$ & 1 \\
\hline Bifurcated & $26 \%$ & $28 \%$ & 0.78 \\
\hline Calcified & $6 \%$ & $6 \%$ & 1 \\
\hline
\end{tabular}

Values are mean $\pm S D$ or $n(\%)$. 


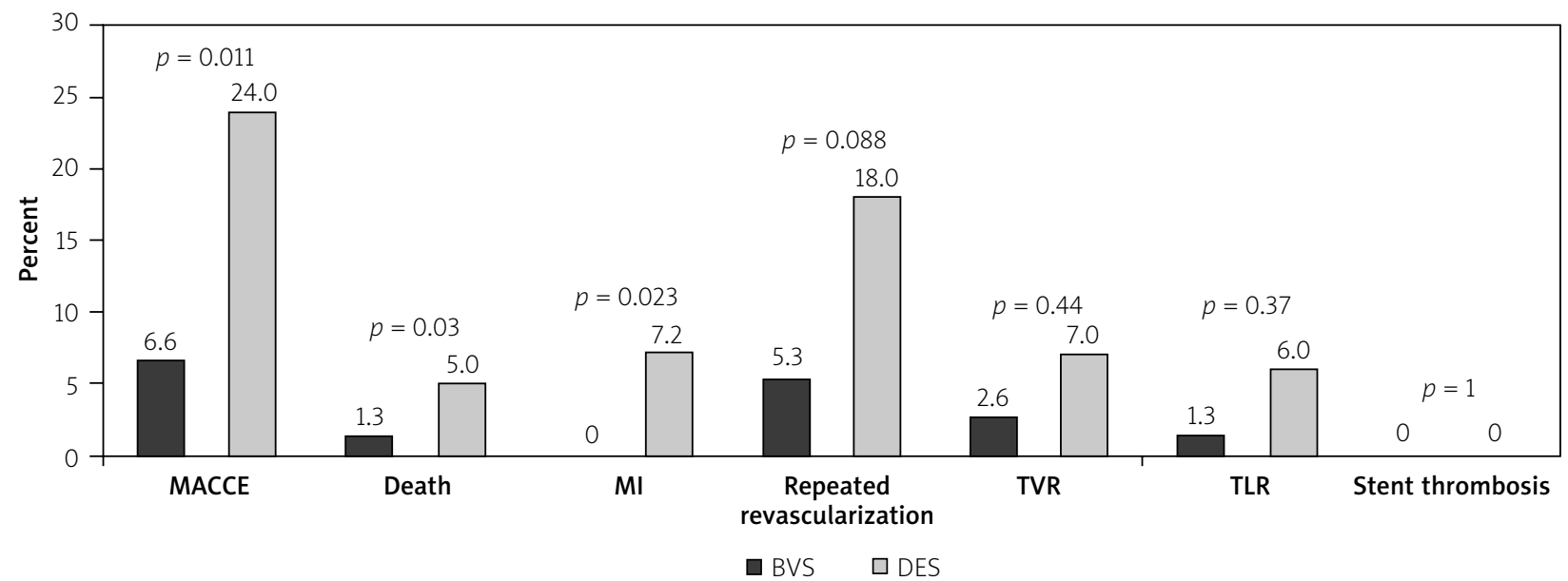

Figure 1. Unadjusted results at 1 year follow-up

BVS - bioresorbable vascular scaffold, DES - metallic drug eluting stent - Xience V.

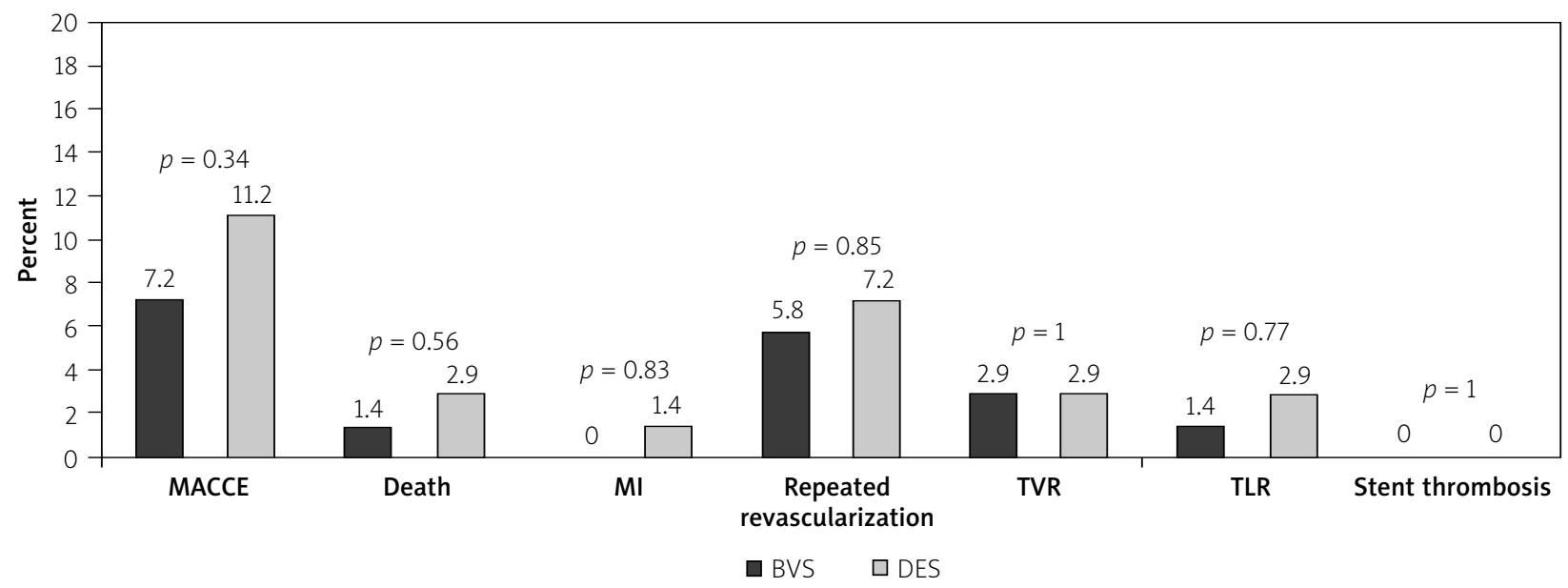

Figure 2. Adjusted results at 1 year follow-up

BVS - bioresorbable vascular scaffold, DES - metallic drug eluting stent-Xience V.

itations that prompt searching for alternative methods to treat coronary artery disease (CAD). Their greatest disadvantage is permanent presence of foreign material in the vessel, which may lead to late adverse events, such as increased risk of very late stent thrombosis ( $>1$ year), acquired stent malapposition, strut fracture or delayed neointimal hyperplasia. It is not uncommon for these events to occur beyond the 5-year period [9].

In this context, BVS technologies seem to be particularly attractive, as theoretically they may allow the vessel to heal and restore its physiological functions over time. These properties may eliminate very late adverse cardiac events (including stent thrombosis). Moreover, biodegradable stents eliminate the concern that some patients have at the thought of having an implant in their bodies for the rest of their lives [10].

Our study, although relatively small, should be regarded as important, as it describes clinical data from a real life patient populations and compares them to the XIENCE V - an everolimus-eluting DES that is well estab- lished on the market. The main finding of our study is that throughout the 1-year follow-up, clinical outcomes of patients who successfully received an Absorb BVS did not differ from those who received a Xience $V$ EES. As expected (due to the retrospective nature of the study) patients' characteristics were slightly different between the groups. Patients who were implanted with a BVS were younger, had higher LVEF, had lower prevalence of multivessel disease and were more likely to be treated in the context of stable angina. In addition, most of the lesions in the BVS group were classified as simple. This reflects the current routine clinical practice, in which physicians tend to select biodegradable technologies for younger patients and for relatively simple clinical scenarios. Despite those discrepancies between studied groups, the propensity score analysis diminished the majority of them, making the registry more reliable. It is important to add that in the BVS group one cardiac death occurred but was classified as not related to the studied device as it occurred due to balloon predilatation. 
A higher prevalence of stable angina and lower rate of multi-vessel disease were also reported by Rzeszutko et al. [11]. Similarly, the main periprocedural major adverse event was coronary artery perforation.

The current study revealed statistically higher pre-dilatation rates for the Absorb BVS as compared to the Xience $\mathrm{V}$. This situation reflects mechanical properties of the polymeric scaffold, which implies careful vessel sizing before the implantation as well as proper vessel preparation. For these reasons the BVS should not be implanted into lesions that cannot be adequately prepared with balloon inflations or when the result of preparation is unsatisfactory [12]. According to the Polish National $\mathrm{PCl}$ Registry, comparable clinical outcomes between the BVS and DES could be achieved independently of vessel tortuosity, calcifications or bifurcation, provided that the lesion is properly prepared [13].

The results of our study are similar to those obtained in other trials that compared Absorb BVS to Xience stents. One such study is Absorb Japan, comprising 400 patients, who were randomized to the Absorb BVS group $(n=266$ patients) or the cobalt-chromium everolimus-eluting stent (CoCr-EES) group ( $n=134$ patients). The primary endpoint of the study was target lesion failure (TLF), defined as a composite of cardiac death, MI attributable to target vessel, or ischemia-driven target lesion revascularization, powered by non-inferiority. At 12-month follow-up there was no significant difference between the two groups in terms of TLF (Absorb BVS $=4.2 \%$ vs. Xience $=3.8 \% ; p$ non-inferiority $<0.0001)$. The secondary end-point evaluated after 13 months was in-segment late lumen loss (LLL), which was $0.13 \pm 0.30 \mathrm{~mm}$ for the Absorb BVS and $0.12 \pm 0.32 \mathrm{~mm}$ for the CoCr-EES ( $p$ non-inferiority $<0.0001$ ). Overall, the study showed comparable clinical results between the Absorb BVS and the CoCr-EES in patients with de novo lesions, with the exception of complex lesions and with an optimal lesion preparation [14].

Another prospective, multicenter, single-blinded, active-treatment controlled clinical trial, ABSORB III, measured the safety and efficacy of BVS compared to DES at one year in patients with CAD. The patients were randomized to receive an everolimus-eluting BVS $(n=1,322)$ or a cobalt-chromium everolimus-eluting stent (CoCr-EES; $n=686)$. Although the primary endpoint of target lesion failure (TLF) showed no difference at 1-year follow-up (7.8\% for Absorb and $6.1 \%$ for Xience; non-inferiority $p<0.007$ ) [15], the recently published 3-year follow-up data presented some worrisome findings. The primary composite endpoint of target lesion failure throughout 3 years of follow-up occurred in $13.4 \%$ of BVS patients and $10.4 \%$ of EES patients $(p=0.06)$, myocardial infarction related to the target vessel increased with BVS (8.6\% vs. $5.9 \% ; p=0.03$ ) and device thrombosis occurred in $2.3 \%$ vs. $0.7 \%(p=0.01)$. In BVS patients treatment of vessels with a reference diameter of less than $2.25 \mathrm{~mm}$ (based on qualitative comparative analysis) was an independent predictor of 3-year TLF and scaffold thrombosis.

In a recently published meta-analysis the Absorb BVS was compared to the metallic EES (Xience; Abbott Vascular; Promus Element; Boston Scientific, Natick, MA, USA) [16]. It included 6 studies, comprising 3738 patients who had Absorb BVS $(n=2337)$ or EES $(n=1401)$ implantation. The results revealed comparable occurrence of TLR as a primary end-point evaluating its efficacy $(p=0.87)$, MI risk $(p=0.06)$ and death $(p=0.89)$ at 12 -month follow-up. However, at the same time, patients with a BVS had higher risk of definite or probable stent thrombosis $(p=0.05)$, particularly during the first 30 days after the procedure $(p=0.02)$.

The similar finding of increased risk of stent thrombosis was also described in the GHOST registry, which revealed cumulative incidence of definite/probable scaffold thrombosis at the level of $1.5 \%$ at 30 days and $2.1 \%$ at 6-month follow-up. All these results should be interpreted in the context of BVS construction limitations and implantation techniques [17]. The Absorb BVS has thick struts with an average thickness of $157 \mu \mathrm{m}$. Compared to other currently used metallic stents, this construction is more thrombogenic and is less forgiving in relation to suboptimal deployment (malapposition, underexpansion, etc.). Thicker struts cause loss of laminar flow and increased platelet activation [18]. In a recently published study thin strut EES revealed decreased platelet aggregation and inflammatory cell adhesion in an animal model as compared to BVS. This finding may explain higher rates of stent thrombosis in BVS, particularly early after stent implantation [19].

Mattesini et al. compared the acute performance of the PLLA Absorb BVS with a second-generation metal DES in complex coronary artery lesions with stent optimization under optical coherence tomography (OCT) guidance. Optical coherence tomography findings between the two groups were not statistically significant, which supports the hypothesis that properly selected patients, systematic aggressive lesion preparation, sizing of a BVS to the proximal reference vessel diameter (RVD), high pressure post-dilation, and use of OCT for optimization enables us to achieve post-procedural area stenosis, minimal lumen area, and an eccentricity index similar to that of contemporary DES platforms that theoretically may decrease the thrombosis rate [20]. Indeed, in the sub-analysis of the Absorb III trial presented by G. Stone, it was found that procedural optimization applied in very thrombogenic arteries of less than $2.25 \mathrm{~mm}$ using high pressure post-dilatation ( $\geq 14 \mathrm{~atm}$ ) was able to significantly decrease the risk of scaffold thrombosis from $8.1 \%$ (all cases with no post-dilatation) to less than 2\% (the rate of stent thrombosis in arteries above $2.5 \mathrm{~mm}$ was $0.8 \%$ and did not differ from Xience stents) [21]. 
Although in our study we did not use intravascular imaging for vessel sizing and implantation optimization, there was no stent thrombosis in either studied group either at short or long-term follow-up. The reason for this unexpectedly favorable result (especially in the light of results presented above) may be a trend toward selecting younger patients and relatively simple clinical scenarios that naturally decrease the risk of stent thrombosis. In addition, in our study we avoided small arteries that by visual estimation were $2.5 \mathrm{~mm}$ or smaller. This may have helped to eliminate the risk of thrombotic events. All patients in the study were prescribed with dual antiplatelet therapy for 12-month follow-up.

\section{Conclusions}

Bioresorbable vascular scaffold is a promising technology that is still at the early stage of development. In our study, which reflects current routine clinical practice for bioresorbable technologies, we found that throughout 1-year follow-up, clinical outcomes of patients who successfully received an Absorb BVS did not differ from those of patients who received a Xience $V$ EES. Long-term follow-up data are required to determine whether these findings will persist beyond 1 year.

\section{Conflict of interest}

The authors declare no conflict of interest.

\section{References}

1. Lüscher TF, Steffel J, Eberli FR, et al. Drug-eluting stent and coronary thrombosis: biological mechanisms and clinical implications. Circulation 2007; 115: 1051-8.

2. Yahagi K, Kolodgie FD, Otsuka F, et al. Pathophysiology of native coronary, vein graft, and in-stent atherosclerosis. Nat Rev Cardiol 2016; 13: 79-98.

3. Lesiak M, Araszkiewicz A. Leaving nothing behind: is the bioresorbable vascular scaffold a new hope for patients with coronary artery disease? Postep Kardiol Interw 2014; 10: 283-8.

4. Kereiakes DJ, Ellis SG, Metzger C, et al. 3-year clinical outcomes with everolimus-eluting bioresorbable coronary scaffolds: the ABSORB III trial. J Am Coll Cardiol 2017; 70: 2852-62.

5. Serruys PW, Chevalier B, Sotomi Y, et al. Comparison of an everolimus-eluting bioresorbable scaffold with an everolimus-eluting metallic stent for the treatment of coronary artery stenosis (ABSORB II): a 3 year, randomised, controlled, single-blind, multicentre clinical trial. Lancet 2016; 388: 2479-91.

6. Thygesen K, Alpert JS, Jaffe AS, et al. Third universal definition of myocardial infarction. Glob Heart 2012; 7: 275-95.

7. Vranckx P, Kint PP, Morel MA, et al. Identifying stent thrombosis, a critical appraisal of the academic research consortium (ARC) consensus definitions: a lighthouse and as a toe in the water. Eurolntervention 2008; 4 (Suppl C): C39-44.

8. The Xience $V$ and Xience Nano Everolimus Eluting Coronary Stent Systems. Instructions for Use. EL2078398. 2015. Abbot Vascular.

9. Park SJ, Kang SJ, Virmani R, et al. In-stent neoatherosclerosis: a final common pathway of late stent failure. J Am Coll Cardiol 2012; 59: 2051-7.
10. Onuma Y, Serruys PW. Bioresorbable scaffold: the advent of a new era in percutaneous coronary and peripheral revascularization? Circulation 2011; 123: 779-97.

11. Rzeszutko L, Tokarek T, Siudak Z, et al. Patient profile and periprocedural outcomes of bioresorbable vascular scaffold implantation in comparison with drug-eluting and bare-metal stent implantation. Experience from ORPKI Polish National Registry 2014-2015. Postep Kardiol Interw 2016; 12: 321-8.

12. Rzeszutko L, Depukat R, Dudek D. Biodegradable vascular scaffold ABSORB BVS ${ }^{T M}$ - scientific evidence and methods of implantation. Adv Interv Cardiol 2013; 9: 22-30.

13. Rzeszutko L, Siudak Z, Tokarek T, et al. Twelve months clinical outcome after bioresorbable vascular scaffold implantation in patients with stable angina and acute coronary syndrome. Data from the Polish National Registry. Adv Interv Cardiol 2016; 12: 108-15.

14. Kimura T, Kozuma K, Tanabe K, et al. A randomized trial evaluating everolimus-eluting Absorb bioresorbable scaffolds vs. everolimus-eluting metallic stents in patients with coronary artery disease: ABSORB Japan. Eur Heart J 2015; 36: 3332-42.

15. Ellis SG, Kereiakes DJ, Metzger DC, et al. Everolimus-eluting bioresorbable scaffolds for coronary artery disease. N Engl I Med 2015; 373: 1905-15.

16. Cassese S, Byrne RA, Ndrepepa G, et al. Everolimus-eluting bioresorbable vascular scaffolds versus everolimus-eluting metallic stents: a meta-analysis of randomised controlled trials. Lancet 2016; 387: 537-44.

17. Capodanno D, Gori T, Nef H, et al. Percutaneous coronary intervention with everolimus-eluting bioresorbable vascular scaffolds in routine clinical practice: early and midterm outcomes from the European multicentre GHOST-EU registry. Eurolntervention 2015; 10: 1144-53.

18. Colombo A, Ruparelia N. Who is thrombogenic: the scaffold or the doctor? Back to the future! JACC Cardiovasc Interv 2016; 9: 25-7.

19. Koppara T, Cheng Q, Yahagi K, et al. Thrombogenicity and early vascular healing response in metallic biodegradable polymer-based and fully bioabsorbable drug-eluting stents. Circ Cardiovasc Interv 2015; 8: e002427.

20. Mattesini A, Secco GG, Dall'Ara G, et al. ABSORB biodegradable stents versus second-generation metal stents: a comparison study of 100 complexlesions treated under OCT guidance. JACC Cardiovasc Interv 2014; 7: 741-50.

21. Stone GW. Expanded Use. TCT Conference Washington. October 29; 2016. https://www.tctmd.com/slide/absorb-bvs-what-havewe-learned 